\title{
ASSISTÊNCIA FARMACÊUTICA MUNICIPAL NA ATENÇÃO À SAÚDE: DESAFIOS PARA A UNIVERSIDADE
}

\author{
Julieta Ueta*, Osvaldo de Freitas*, Ajith Kumar Sankarankutty**, \\ Eduardo Barbosa Coelho **, Luciane Loures dos Santos ${ }^{* * *}$, Sonia Mara Neves Ferri ${ }^{* * *}$
}

\section{RESUMO}

O acesso universal e gratuito, de qualidade e racional a serviços e a medicamentos pelo SUS tem garantia constitucional. Os medicamentos, ferramenta essencial para a saúde, são também responsáveis por elevados índices de morbimortalidade. Criação de estratégias para o aprimoramento das práticas de acadêmicos e profissionais da área de saúde sobre o uso racional de medicamentos tem sido a meta do Programa de Educação para o Uso Racional de Medicamentos PURAME. Trata-se de um projeto de Extensão Universitária, entendendo-a como "o processo educativo, cultural e científico que articula o Ensino e a Pesquisa de forma indissociável e viabiliza a relação transformadora entre Universidade e Sociedade”. 0 estudo foi desenhado para desenvolver ações que resultem na segurança na utilização de medicamentos, prevenindo e reduzindo seus problemas, com a participação de acadêmicos e profissionais de saúde. Campanhas educativas, cursos sobre medicamentos, gerenciamento de medicamentos e práticas clínicas farmacêuticas foram realizadas em Ribeirão Preto e Luiz Antonio resultando em treinamento de acadêmicos e profissionais, bem como orientação de usuários sobre o uso correto de medicamentos. Além do mais, práticas clínicas de farmácia reduzem índices de morbimortalidade causada por medicamentos. A implementação destas ações e práticas através de acadêmicos insere a Universidade na realidade e democratiza conhecimentos que resultam em melhor qualidade de vida.

Palavras-chave: Assistência farmacêutica. Medicamentos. Extensão Universitária.

\section{ABSTRACT}

The Brazilian universal Healthcare system (SUS) by constitution free universal access to high quality and rational services and medications, essential tool for health are also responsible for high morbidity and mortality rates.The development of strategies to improve practices of healthcare students and professional on rational medication use has been the goal of the Education Program for the Rational Medication Use (PURAME). It deals with university Extensions project, understanding it as "the education, cultural or cientific process that articulates Teaching and Research in non dissociable way and makes possible the transforming relationship between University and Society”. The study was drawn in order to develop actions that result in medication safety, preventing and reducing their problems, with the students and professionals participation. Educating campaigns, course about medications, manegement and clinical pharmacy pratices are developed in Ribeirão Preto and Luiz Antonio municipalities resulting in students and professionals training, as well as patients counseling on correct medications use. Besides, clinical pharmacy practices reduce morbidity and mortality rates caused by medications. The implemention of these actions and practices through students inserts the university in the reality and democratizes knowledge that result in better quality of life.

Key words: Pharmaceutical assistance. Medications. Extension activities.

* FCFRP-USP ** FMRP-USP. ***Docente FAEPA-FMRP-USP. 


\section{INTRODUÇÃO}

Medicamentos são essenciais para os indivíduos e para os serviços de saúde por reduzirem os índices de morbimortalidade e melhorarem a qualidade de vida das pessoas. $\mathrm{O}$ acesso aos medicamentos e aos serviços de saúde e sua qualidade são cada vez mais incorporados pela sociedade como um bem fundamental e um direito humano. Os medicamentos salvam vidas, reduzem sofrimento e melhoram as condições de saúde se forem de qualidade e se devidamente bem utilizados. Segundo a Organização Mundial de Saúde, a utilização de medicamentos compreende a aquisição, a distribuição, a prescrição, a dispensação e o uso pelos pacientes de medicamentos em uma sociedade, enfatizando especialmente as consequências médicas, sociais e econômicas que dela resultam (WHO, 1977). A meta dos serviços de saúde deve ser a promoção da utilização racional dos medicamentos (WHO, 1994; COHEN, 2000, MANASSE, 1989 a,b). A qualidade da atenção à saúde do indivíduo depende, portanto, de uma assistência farmacêutica de qualidade, ou seja, da qualidade do acesso e da utilização racional dos medicamentos (BATES et al., 1998).

Com a promulgação da Constituição Federal em 1988 foi estabelecido que a saúde é um direito da sociedade e o cuidar da saúde ficou sob a competência das três esferas do Governo (União, estados e municípios) de acordo com os artigos 6ㅇ e 23. 0 artigo 196 determina que "A saúde é direito de todos e dever do Estado, garantido mediante políticas sociais e econômicas que visem à redução do risco de doença e de outros agravos e ao acesso universal e igualitário às ações e serviços para sua promoção, proteção e recuperação" (BRASIL, 1988).

A Lei Orgânica da Saúde (8080/90) em seu artigo $6^{\circ}$ determina a formulação da Política de Medicamentos e atribui ao setor da saúde a responsabilidade pela execução "de ações de assistência terapêutica integral, inclusive farmacêutica.” Desta forma, fica claro que a assistência farmacêutica é considerada parte integrante da atenção à saúde.

A concepção de Assistência Farmacêutica varia consideravelmente entre os países desenvolvidos e em desenvolvimento, englobando princípios básicos que norteiam o papel do farmacêutico e de outros profissionais no sistema de atenção à saúde. Para o Brasil, o termo Assistência Farmacêutica envolve atividades de caráter abrangente, multiprofissional e intersetorial, que situam como objeto de seu trabalho a organização das ações e serviços relacionados ao medicamento em suas diversas dimensões, enfatizando a interação com o paciente e a comunidade na visão da promoção da saúde (MARIN et al., 2003).

Em 30 de outubro de 1998 foi aprovada a Política Nacional de Medicamentos (PNM) através da Portaria GM/MS n. 3916 (BRASIL, 1998).

A assistência farmacêutica é definida na Política Nacional de Medicamentos (BRASIL, 1998) como "Grupo de atividades relacionadas com o medicamento, destinadas a apoiar as ações de saúde demandadas por uma comunidade. Envolve o abastecimento de medicamentos em todas e em cada uma de suas etapas constitutivas, a conservação e o controle de qualidade, a segurança e a eficácia terapêutica dos medicamentos, o acompanhamento e a avaliação da utilização, a obtenção e a difusão de informação sobre medicamentos e a educação permanente dos profissionais de saúde, do paciente e da comunidade para assegurar o uso racional de medicamentos".

A estruturação da Assistência Farmacêutica é um dos grandes desafios para gestores e profissionais do SUS, quer pelos elevados e parcos recursos financeiros envolvidos como pela necessidade de aperfeiçoamento contínuo, com busca de novas estratégias no seu gerenciamento.

No SUS, estabeleceram-se a universalidade, a equidade e a integralidade como princípios éticos/doutrinários e a descentralização, a regionalização, a hierarquização e controle social como seus princípios. Portanto, o acesso universal e gratuito a serviços públicos e a medicamentos padronizados pelo SUS tem garantia constitucional.

As ações não se restringem apenas à aquisição e distribuição de medicamentos exigindo, para a sua implementação, a elaboração de planos, programas e atividades específicas, de acordo com as competências estabelecidas para cada esfera de governo. Aos gestores cabe 0 aperfeiçoamento e a introdução de novas estratégias que garantam a eficiência de suas ações junto aos serviços de saúde e junto à população e que resultem na garantia do acesso aos medicamentos de qualidade e sua utilização racional, visto que os medicamentos, ferramenta essencial para a saúde, são também responsáveis por elevados índices de morbimortalidade.

Os riscos, danos e custos do uso irracional de medicamentos podem ser imensos para a sociedade 
contrapondo-se ao conceito da "pílula milagrosa" em benefício da saúde do homem. Diariamente, em todo o mundo, a saúde das pessoas está sendo prejudicada pelo uso incorreto de medicamentos. Fatores como a falta de informações claras e objetivas sobre os medicamentos para aqueles que os prescrevem e para aqueles que os consomem influencia a escolha dos medicamentos e encoraja as pessoas a usá-los inadequadamente, em situações, talvez, desnecessárias. A OMS estima que nos países em desenvolvimento o número de intoxicações atinge $3-4 \%$ da população. Dados do Food and Drug Administration (FDA) mostraram que cerca de $20 \%$ dos relatos de reações adversas que poderiam ser evitados levaram à hospitalização ou morte (MANASSE 1989 a,b; JOHNSON \& BOOTMAN, 1995, 1997; CASSIANI \& UETA, 2004).

Os altos índices de morbidade e mortalidade decorrentes da prática inapropriada dos profissionais no trato com os medicamentos têm alarmado as autoridades sanitárias, pesquisadores e profissionais de saúde. Os estudos realizados, quase que exclusivamente em ambientes hospitalares, permitiram caracterizar os erros na medicação e os problemas relacionados com os medicamentos. Os erros estão relacionados à prática profissional, aos procedimentos e sistemas, incluindo: prescrição, transcrição, requisição, identificação do produto, embalagem, nomenclatura, composição, dispensação distribuição, administração do medicamento, educação, monitoramento e uso (ISMP Institute of Safe Medication Practices; PHILLIPS et al., 2001; JOHNSON \& BOOTMAN, 1995).

No relatório de 1999 "To Err Is Human" do Institute of Medicine (1999) foi descrito que 7000 mortes por ano são consequência de erros na medicação, enquanto reações adversas a medicamentos podem ser responsáveis por cerca de 100.000 mortes a cada ano, correspondendo da $4^{-}$a $6^{-}$causa de morte nos Estados Unidos (INSTITUTE MEDICINE, 1999). Morbidade e mortalidade relacionados com os medicamentos têm o custo estimado entre 76,6 e 136 bilhões de dólares anuais (JOHNSON \& BOOTMAN, 1997).

Um estudo realizado em 1116 hospitais americanos e a avaliação de 430.586 relatos de erros na medicação que resultaram em algum dano para o paciente mostrou que estes aconteceram em 5,07\% dos pacientes admitidos a cada ano nestes hospitais; um evento a cada 22,7 horas (um a cada 19,73 admissões); com a ocorrência em 3-6.9\% dos internados; $11 \%$ de- les decorreram de erros na dispensação pela farmácia, seja na medicação ou na dose errada. Os fatores responsáveis pelo aumento significativo de erros na medicação foram ocasionados por falta de vínculo com estabelecimento de ensino farmacêutico, pela centralização dos farmacêuticos na Farmácia Central, pelo reduzido número de enfermeiros registrados e de farmacêuticos por leito ocupado (BOND et al., 2001).

Há um círculo vicioso que opera no uso indevido: os recursos que poderiam ser empregados diretamente na prevenção e controle das doenças são desviados e usados para tratar as consequências deste uso indevido. Estudos sobre as causas dos erros na medicação têm apontado para falhas na comunicação (prescrições manuscritas como mostrado em LESAR et al.,1997), nomes similares de medicamentos, pontos decimais e zeros, abreviações e solicitações ambíguas e incompletas), sistemas de distribuição inadequados, erros de cálculo, administração incorreta e falta de orientação do paciente (COHEN, 2000).

$\mathrm{Na}$ tentativa de minimizar os erros, os pesquisadores têm procurado desenvolver procedimentos e sistemas, como a padronização de medicamentos, o sistema informatizado de prescrição (CPOE - computorized physician order entry), a informatização de informações para suporte à decisão clínica, o sistema de identificação por código de barras, além da presença do farmacêutico em áreas clínicas e o trabalho em equipe (BATES et al., 1998; CASSIANI \& UETA, 2004).

Os estudos mostram que eventos adversos são, em geral, evitáveis se práticas de racionalidade e de segurança (medication safety) forem empregadas pelos profissionais de saúde, melhorando a qualidade e custos da terapia. Os idosos são os maiores consumidores dos medicamentos e ficam mais sujeitos às reações e aos eventos adversos agravados pela susceptibilidade do quadro fisiopatológico do envelhecimento.

A ciência da implementação em saúde tem como foco promover a captação e transferência de conhecimentos gerados nos meios acadêmicos para os serviços e sistemas de saúde. Estes conhecimentos estão relacionados a intervenções baseadas em evidências que comprovadamente aprimoram a qualidade da assistência à saúde. Novos conhecimentos são gerados no processo de inserção de novas tecnologias e conhecimentos, tendo sido criada uma nova área de estudo conhecida como a ciência da implementação. 
A ciência da implementação é o estudo científico de métodos para promover a captação de achados científicos, e outras práticas baseadas em evidências, em prática rotineira e, assim, melhorar a qualidade e a efetividade dos serviços e cuidados da saúde. Este campo relativamente novo inclui, também, o estudo de influências sobre os profissionais de saúde e o comportamento organizacional.

Trata-se, portanto, de introduzir e entender como a sociedade pode usufruir do conhecimento de alta qualidade. Se esta ação vinculada ao ensino e à busca de novos conhecimentos for realizada pela Universidade pode-se afirmar que se trata de uma atividade de Extensão. Sem fragmentação, livre da afirmação de que existe um tripé para o papel da Universidade, pode-se entender que esta ação estabeleça uma prática acadêmica que leva e traz conhecimentos pela interação com o serviço, ou seja, com a sociedade (BOTOMÉ, 2002) onde acadêmicos, profissionais de saúde e usuários do serviço podem, se bem sucedidos, usufruir do aprimoramento das práticas de assistência à saúde, estabelecendo-se um processo de contínuo aprendizado, com geração de novos conhecimentos resultante da inserção social da Universidade através de graduandos, pós-graduandos e docentes.

A criação de estratégias para o aprimoramento das práticas de acadêmicos da área de saúde e profissionais médicos, de enfermagem, farmacêuticos e agentes comunitários de saúde, e a educação da população e orientação de indivíduos sobre o uso racional de medicamentos, tem sido a meta do Programa de Educação para o Uso Racional de Medicamentos - PURAME.

Este projeto do PURAME voltado à assistência farmacêutica municipal teve como objetivo desenvolver práticas que resultem na segurança na utilização de medicamentos, prevenindo e reduzindo problemas com os medicamentos, e educação em saúde através de campanhas educativas em praças públicas e educação permanente de acadêmicos, profissionais de saúde. Pretendeu-se desenvolver ações e atividades reconhecidas como promotoras da segurança dos pacientes para se consolidar modelo acadêmico nos serviços municipais de saúde, onde o usuário dos serviços será o beneficiário das ações e atividades.

\section{DELINEAMENTO DO ESTUDO}

Trata-se de um projeto de Extensão desenvolvido no período de março de 2007 a abril de 2008 com a introdução de práticas de acesso e utilização racional de medicamentos nos municípios de Ribeirão Preto (Distrito Oeste: serviços coordenados pela FMRP-USP-SP) e de Luiz Antonio-SP.

\section{A. DADOS DOS MUNICÍPIOS}

1. Distrito Oeste de Ribeirão Preto tem cerca de 170 mil habitantes, com uma UBDS, várias UBS e cinco Núcleos de Saúde de Família (NSF), com uma equipe em cada NSF;

2. Luiz Antônio, município a $60 \mathrm{~km}$ de Ribeirão Preto tem segundo o IBGE 7994 habitantes e 10.092 de acordo com o SIAB (Sistema de Informação de Atenção Básica). Cerca de 98,8\% da área é rural, onde vivem 717 habitantes do total do SIAB. Na área urbana há água encanada, coleta de lixo e tratamento de esgoto, mas a realidade da área rural é outra. Tem uma Unidade Mista de Saúde que funciona 24h, com pronto atendimento e especialidades e três equipes de Saúde da Família.

\section{B. AÇÕES/ATIVIDADES DESENVOLVIDAS}

1. Campanhas educativas em diversos locais públicos: praça XV de Novembro, Ribeirão Preto; UBS Dutra, Bairro Dutra-RP; Núcleo de Saúde da Família 3 (NSF-3); Unidade Mista de Saúde de Luiz Antônio-SP;

2. Ministração de cursos de utilização racional de medicamentos de acordo com modelos da OMS para acadêmicos de medicina, farmácia e outros cursos e do Departamento de Assistência Farmacêutica (DAF) do Ministério da Saúde para equipes de saúde da família, principalmente agentes comunitários de saúde;

3. Gerenciamento de medicamentos: aquisição e organização de medicamentos obtidos por doação; acesso aos medicamentos;

4. Práticas de Farmácia Clínica: desenvolvimento de um sistema informatizado para o processo de prescrição, atendimentos farmacêuticos com seguimento farmacoterapêutico para detecção e solução de problemas relacionados aos medicamentos; avaliação de prescrições de banco de dados, criação de Comissão de Medicamentos e Terapêutica. 
Todas as ações e atividades fizeram parte do processo de aprimoramento da assistência farmacêutica no município de Luiz Antônio-SP.

\section{PÚBLICO-ALVO}

Das instituições de ensino e dos serviços de saúde:

1. Acadêmicos do 5을 ano do curso de medicina (200) e de farmácia (240);

2. Profissionais de saúde: médicos, outros profissionais de saúde, residentes, agentes comunitários de saúde (160).

Usuários dos serviços de saúde:

1. Área de abrangência de quatro equipes de saúde de família em Ribeirão Preto: até 4000 famílias;

2. População de Luiz Antônio: 10 mil habitantes, 7200 dispensações/mês na Farmácia da Unidade Mista de Saúde;

3. Campanhas na praça: até 1500 pessoas por campanha.

\section{RESULTADOS}

As ações desenvolvidas pelo PURAME foram reunidas em quatro grupos: campanhas educativas, cursos sobre medicamentos, gerenciamento de medicamentos e práticas de farmácia clínica.

Os resultados obtidos estão apresentados nos Quadros 1 a 4 organizados por ações, atividades pertinentes realizadas e resultados alcançados.

No Quadro 1 estão descritas as campanhas educativas, os locais e o pessoal e público envolvidos. A Campanha " 5 de maio" tornou-se uma tradição nacional com nucleação inicial na praça central de Ribeirão Preto, com a participação de bolsistas do PURAME, apoiados pela COSEAS, e pelo Fomento às Iniciativas de Cultura e Extensão (antigo Fundo de Cultura e Extensão Universitária). Os bolsistas participavam da elaboração de material educativo, da organização das campanhas e da realização das atividades da campanha. 0 trabalho junto às unidades de saúde resultou em sucesso, onde os indivíduos com problemas de saúde, ou com os medicamentos, receberam orientação e encaminhamento para os serviços para consultas, se necessário. Os acadêmicos do PURAME enriqueceram sua relação com as pessoas, aprimoraram seus conhecimentos e aprenderam a organizar as atividades, antes e durante a campanha.
No Quadro 2 estão descritas a ministração de cursos sobre uso racional de medicamentos. Os cursos ministrados aos acadêmicos e profissionais de saúde com nível superior foram adaptados da proposta da Organização Mundial de Saúde, com coordenação da ANVISA. A capacitação de ministrantes foi realizada em Águas de São Pedro-SP e em Ribeirão Preto-SP (WANNMACHER, 2005; PETRAMALE, 2004).

0 curso de utilização racional de medicamentos para ACS foi ministrado a agentes comunitários de saúde. Segundo Marques (2008), os ACS treinados para a utilização racional de medicamentos não apresentaram nível de conhecimento maior que os não treinados, de acordo com o instrumento de avaliação utilizado pela autora.

No Quadro 3 estão apresentados dados e os resultados do gerenciamento dos medicamentos. Os medicamentos que são distribuídos pelos representantes das empresas farmacêuticas para os prescritores dos núcleos de saúde da família são organizados pelos estagiários bolsistas para adequar a disponibilidade e para o aprendizado sobre as diferentes classes de medicamentos. Estoques são organizados e mantidos no laboratório de Assistência Farmacêutica da FCFRPUSP para serem disponibilizados para a população atendida na área. O PURAME também se responsabiliza para possibilitar o acesso das pessoas aos medicamentos que elas necessitam, orientando prescritores e os usuários de medicamentos.

As práticas de farmácia clínica se traduzem em atenção farmacêutica onde se procura definir um plano de acompanhamento dos usuários do serviço que apresentam problemas com o uso de medicamentos, seja por falta de acesso, dificuldades de adesão ou de uso correto, livre de interações e reações adversas. Também constitui parte da prática clínica da farmácia a análise de prescrições. No Quadro 4 estão apresentadas as atividades desenvolvidas e os resultados obtidos (UETA et al., 2005).

Com base nas intervenções sugeridas pela Organização Mundial de Saúde (WHO, 2002 e 2003) foram introduzidas ações junto aos serviços de saúde do município de Luiz Antônio. Criou-se um banco de dados com lista de medicamentos dispensados a pacientes especificados com data para se realizar uma análise gerencial, bem como uma análise das práticas de prescrições, definindo-se o perfil de prescrição de cada prescritor. 
Obtiveram-se os dados referentes às prescrições inadequadas, que foram empregados para se discutir com cada prescritor alternativas mais eficazes, seguras e custo efetivas. Foi elaborada uma sugestão de lista padronizada de medicamentos para os prescritores opinarem e criada uma Comissão de Medicamentos e Terapêutica que deliberava sobre as questões de acesso e utilização racional de medicamentos (UETA et al., 2005 b).

A adoção de sistemas informatizados de prescrição é considerada um mecanismo de segurança do paciente por reduzir significantemente os erros. Foi desenvolvido um software para prescrição, com suporte à decisão clínica, financiado pelo CNPq para ser disponibilizado para as unidades de saúde.

\section{DISCUSSÃO}

Este projeto de extensão universitária foi desenvolvido com a visão de segurança do paciente: "não cause danos, não acuse”, desenvolva sistemas mais seguros. A segurança no processo de utilização de medicamentos requer prescritores e profissionais de saúde melhor treinados e informações melhor disponibilizadas para permitir tomada de decisões mais corretas para os usuários dos serviços. Pretendeu-se trabalhar com ciência da implementação, inserindo práticas consolidadas na rotina de serviços através do contato direto dos acadêmicos com os usuários, ou através do treinamento dos acadêmicos com as melhores práticas de utilização dos medicamentos. 0 desenvolvimento do projeto possibilitou aos acadêmicos participantes a percepção da realidade dos serviços e o contato com a população em um processo de troca de saberes.

Atividades de extensão universitária são entendidas como atividades desenvolvidas pela universidade junto à comunidade, ou melhor, são atividades que envolvem a universidade e a comunidade. 0 papel da extensão vem se modificando e alguns documentos têm demonstrado os reflexos destas mudanças como a Carta de Florianópolis (2002).

Segundo o Plano Nacional de Extensão (MINISTÉRIO DA EDUCAÇÃO, 2001) “A Extensão Universitária é o processo educativo, cultural e científico que articula o Ensino e a Pesquisa de forma indissociável e viabiliza a relação transformadora entre Universidade e Sociedade. A Extensão é uma via de mão-dupla, com trânsito assegurado à comunidade acadêmica, que encontrará, na sociedade, a oportunidade de elaboração da práxis de um conhecimento acadêmico.” No retorno à universidade, docentes e discentes trarão um aprendizado que, submetido à reflexão teórica, será acrescido àquele conhecimento. Esse fluxo, que estabelece a troca de saberes sistematizados, acadêmico e popular, terá como consequências a produção do conhecimento resultante do confronto com a realidade brasileira e regional, a democratização do conhecimento acadêmico e a participação efetiva da comunidade na atuação da universidade. O objetivo da Extensão Universitária é reafirmar o processo acadêmico definido e efetivado em função das exigências da realidade, indispensável na formação do aluno, na qualificação do professor e no intercâmbio com a sociedade, o que implica em relações multi, inter ou transdisciplinares e interprofissionais.

A ideia de que através do ensino se transmite 0 conhecimento da pesquisa se gera e da extensão se socializa o conhecimento é uma maneira tradicional de se entender o papel da universidade. Pode-se entender que à universidade cabe a geração do conhecimento, seja ela proveniente do ensino, da pesquisa ou da extensão, ou seja, o conhecimento pode ser gerado nos três segmentos ou na integração dos três segmentos.

Nas atividades de extensão, ao se socializar o conhecimento gerado na academia, ocorre a interação com o conhecimento popular e a apropriação deste saber pode conduzir a transformações que consolidam em mudanças da realidade social. A introdução desta nova realidade no cotidiano do ensino e da pesquisa abre perspectivas para a solução dos problemas que se apresentam nas comunidades. 0 conhecimento gerado pela academia, seja ele básico ou aplicado, pode ou não alcançar a sociedade ou com ela contribuir através da transformação. A importância que as instituições de ensino dão a estas atividades, às vezes se traduzem em políticas para as atividades de extensão de universidades (UNESC, 2004; PUC MINAS, 2006).

Os avanços na área da saúde dependem da transferência de conhecimentos gerados pela academia, para evoluir, aprimorar a qualidade da atenção, os resultados terapêuticos, reduzir ou mesmo prevenir morbimortalidade. Serviços de saúde vinculados às instituições de ensino apresentam melhores resultados terapêuticos (BOND et al., 2001). As instituições de ensino da área de saúde realizam atividades de extensão através da inserção de 
alunos graduandos e graduados e docentes junto aos serviços assistenciais de saúde. Neste processo procurase introduzir as melhores práticas, hoje ditas baseadas em evidências clínicas, na rotina dos serviços, privilegiando os usuários com os avanços e conquistas científicas, sejam tecnologias dependentes de equipamentos ou de comunicação entre as pessoas. Atualmente, esforços têm sido despendidos no sentido de se trabalhar com segurança do paciente, na tentativa de reduzir os problemas causados pelos erros humanos (FEDERICO, 2004).

A transferência do conhecimento é um processo de duas vias ou duas mãos: aprende-se ensinando ou ensina-se aprendendo, o que resulta em novos conhecimentos. Na implementação de práticas bem sucedidas nas rotinas dos serviços, o pesquisador depara-se com novas realidades que requerem um reposicionamento. Briceno Leon (1996) retrata o processo de educação sanitária com a elaboração de sete teses, uma delas que diz: "a ignorância não é um vazio a ser preenchido, mas um cheio a ser transformado" e a outra diz: "não há um que sabe e outro que não sabe, mas dois que sabem coisas diferentes".

Com o desenvolvimento deste projeto foram realizadas as campanhas educativas, os cursos sobre utilização racional de medicamentos e as práticas de farmácia clínica que não fazem parte do cotidiano das atividades curriculares dos alunos de farmácia, medicina ou outros. O treinamento de profissionais do serviço, sejam prescritores ou agentes comunitários de saúde, faz parte do processo de educação continuada, instrumento fundamental para a qualidade da assistência à saúde.

O PURAME mantém suas atividades junto aos serviços de saúde dos municípios que solicitam avaliação e implementação de práticas para o aprimoramento da assistência farmacêutica e mantém o foco na segurança do paciente (GANDHI et al., 2004), principalmente, segurança no processo de utilização de medicamentos, como parte da qualidade da assistência à saúde, com a participação acadêmica.

AGRADECIMENTOS: a todos os alunos bolsistas do FomentoPRCEU-USP, da COSEAS-USP, estagiários do PURAME, os acadêmicos dos cursos de graduação do campus da USP em Ribeirão Preto, profissionais de saúde das unidades de saúde que participaram das ações e atividades realizadas pelo PURAME.

\section{REFERÊNCIAS BIBLIOGRÁFICAS}

BATES, D. W.; et al. Effect of computerized physician order entry and a team intervention on prevention of serious medication errors. JAMA, v. 280, p. 1311-1316, 1998.

BOND, C.A; RAEHL, C.L.; FRANKE, T. Medication Errors in United States Hospitals. Pharmacotherapy, v. 21(9), p. 1023-1036, 2001.

BOTOMÉ, SP. A extensão universitária: é necessário superar equívocos, identificar exigências, definir prioridades e ampliar perspectivas para a universidade. Anais do IX Encontro Nacional de Extensão e Ação Comunitária. 2002, Florianópolis, p.71-103. Disponível em apud: <http://www. unesc.rct-sc.br/extensao/docs/20051/politicas.doc>. Acesso em: 12 fev. 2009.

BRASIL, 1988, A constituição do Brasil. Disponível em: <http:// www.planalto.gov.br/ccivil_03/constituicao/Constituiçao_ Compilado.htm>. Acesso em: 19 abr. 2009.

BRASIL, PNM Política Nacional de Medicamentos. 1998. Disponível em: <http://www.opas.org.br/medicamentos/site/ UploadArq/pnm.pdf > . Acesso em: 12 abr. 2009.

BRICENO LEON, R. Siete tesis sobre la educación sanitaria para la participación comunitária. Cadernos de Saúde Pública. Rio de Janeiro, 12(1):7-30, jan-mar, 1996.

CARTA DE FLORIANÓPOLIS. 2002. Disponível em: <http://www. uniso.br/forext/docs/cartas/carta_florianopolis.doc $>$. Acesso em: 19 abr. 2009.

CASSIANI, SHB; UETA, J. A segurança do paciente na utilização da medicação. Ed. Artes Médicas, 2004.

COHEN, M.R. Preventing medication errors related to prescribing. In: Medication errors causes, prevention, and risk manangement. Jones and Bartlett Publishers, MA USA pp. 8.1-8.23, 2000.

FEDERICO, F. Recomendações para as Melhores Práticas da Medicação - Coalizão para a Prevenção de Erros Médicos de Massachusetts - EUA. In: A Segurança dos Pacientes na Utilização da Medicação. UETA, J.; CASSIANI, S. H. B (Eds.), São Paulo, Editora Artes Médicas Ltda, 2004, c. 2, pp. 11-20.

GANDHI, T. K.; KAUSHAL, R.; BATES, D. W. Introdução à Segurança do Paciente. In: A Segurança dos Pacientes na Utilização da Medicação. UETA, J.; CASSIANI, S. H. B. (Eds.), São Paulo, Editora Artes Médicas Ltda., 2004, c. 1, pp. 1-10.

INSTITUTE OF MEDICINE. To err is human. Building a safer health system. Washington, DC: National Academy Press, 1999.

ISMP - Institute for Safe Medication Practices. Disponível em: <http://www.ismp.org>. Acesso em: 01 abr. 2009.

JOHNSON, J.A.; BOOTMAN, J. L. Drug-related morbidity and mortality: A cost-of-illness model. Arch Int Med. V. 155, p. 1949-1956, 1995. 
Drug-related morbidity and mortality and the economic impact of pharmaceutical care. Am J Health Syst Pharm. V. 54:554-8, 1997.

LESAR, T S; BRICELAND, L; STEIN, DS. Factors related to errors in medication prescribing. JAMA, v. 277, p. 312-317, 1997.

MANASSE, H. R. Jr. Medication use in an imperfect world, I: drug misadventuring as an issue of public policy. American Journal Hospital Pharmacy. V. 46, p. 929-944 (a), 1989.

Medication use in na imperfect world, II: drug mis adventuring as an issue of public policy. American Journal Hospital Pharmacy. V. 46, p. 1141-1152 (b), 1989.

MARIN N.; LUIZA V.L.; CASTRO C.G.S.O.; SANTOS S.M. (org). Assistência Farmacêutica para Gerentes Municipais. 2003. Disponível em: <http://www.opas.org.br/medicamentos/index.cfm?ent=2\&carregar=1\#>. Acesso em: 19 out. 2005.

MARQUES, T. As atividades de agentes comunitários de saúde e a promoção do uso correto de medicamentos em unidades do Distrito de Saúde Oeste de Ribeirão Preto - SP, 2008. Disponível em: <http://www.teses.usp.br/teses/disponiveis/22/22132/ tde-06102008-132626/>. Acesso em: 20 fev. 2009.

Ministério da Educação. Secretaria de Educação Superior. Plano Nacional de Extensão 1999-2001. Disponível em: <http://www. mec.gov.br/Sesu/planonaex.shtm>. Acesso em: 22 fev. 2009.

PETRAMALE, C. Ensino para o uso racional de medicamentos, 2004. Disponível em: <http://www.cori.unicamp.br/foruns/ saude/evento15/clarice.ppt\#316,1>. ENSINO PARA O USO RACIONAL DE MEDICAMENTOS. Acesso em: 15 abr. 2009.

PHILLIPS J.; BEAM S.; BRINKER A. et al. Retrospective analysis of mortalities associated with medication errors. Am J Health Syst Pharm.; v. 58, p. 1835-41, 2001.

PUC MINAS. Política de Extensão da PUC Minas, 2006. Disponível em: <http://www.pucminas.br/proex//hotsite/3encontro/ politica.doc $>$. Acesso em: 12 abr. 2009.

UETA J.; SILVA JR D. B.; SANKARANKUTTY A.; DIPE F. Atividades de Farmácia Clínica e de Prescrição para Residentes e Acadêmicos de Medicina. In: I Congresso Paulista de Medicina de Família e Comunidade, 2005. Ribeirão PretoSP. Anais do I Congresso Paulista de Medicina de Família e Comunidade; v. 1, p. 1.6.4 (a), 2005.

UETA J.; SILVA JR D. B.; FERRI S. N. Reorganização da Assistência Farmacêutica em Município do Estado de São Paulo. In: I Congresso Paulista de Medicina de Família e Comunidade, 2005. Ribeirão Preto-SP. Anais do I Congresso Paulista de Medicina de Família e Comunidade; v. 1, p. 1.6.5. (b), 2005.

UNESC. Política de Extensão Universitária da UNESC, 2004. Disponível em:<www.unesc.rct-sc.br/extensao/docs/20051/ politicas.doc>. Acesso em: 15 mar. 2009.

WANNMACHER, L. Uso racional de medicamentos. Disponível em: <http://www.anvisa.gov.br/servicosaude/hsentinela/uso_ racional_I/Lenita_Cursos.ppt\#312,5,Cursos> 2005, sobre
Ensino para Uso Racional de Medicamentos. Acesso em: 15 abr. 2009.

WHO - World Health Organization. Introduction to Drug Utilization Research. Printed in Oslo, Norway, 2003. Disponível em: <http://www.who.int/medicine>. Acesso em: 19 out. 2008.

Selection and Rational Use of Medicines, 2002. Disponível em: <http://www.who.int/medicines/areas/rational_ use/en/index.html>. Acesso em: 19 out. 2008.

WHO/PHARM/DAP/94.12 - Guidelines for good prescribing, 1994. Disponível em: http://whqlibdoc.who.int/ hq/1994/WHO_DAP_94.11.pdf. Acesso em: 19 out. 2005.

The selection of essential drugs. TECHN REPSER, p. 615, Geneva, 1977. 
QUADRO 1

Campanhas educativas realizadas em praças públicas ou unidades básicas de saúde no município de Ribeirão Preto

\begin{tabular}{|c|c|c|c|}
\hline TIPO DE AÇÃO & AÇÃo & ATIVIDADES & RESULTADOS \\
\hline \multirow[t]{9}{*}{$\begin{array}{l}\text { Campanhas } \\
\text { educativas }\end{array}$} & $\begin{array}{l}\text { Campanha } \\
5 \text { de Maio }\end{array}$ & $\begin{array}{l}\text { - Foi ministrada uma aula, dentre outras, } \\
\text { sobre "obesidade e fatores de risco } \\
\text { cardiovascular", incluída nas atividades } \\
\text { de preparação dos alunos do curso de } \\
\text { farmácia da FCFRP-USP para atuarem na } \\
\text { campanha "5 de maio". }\end{array}$ & $\begin{array}{l}\text { - Envolvimento da população de Ribeirão } \\
\text { Preto. Grande número de testes de } \\
\text { glicemia, tipagem sanguínea, aferição de } \\
\text { pressão arterial e cálculo do índice de } \\
\text { massa corpórea (IMC) foram realizados. } \\
\text { Dúvidas das pessoas sobre medicamentos } \\
\text { sanadas durante a campanha. }\end{array}$ \\
\hline & & $\begin{array}{l}\text { - Durante a campanha - orientação } \\
\text { dos alunos e da população sobre } \\
\text { obesidade, hipertensão, diabetes e sobre } \\
\text { medicamentos. }\end{array}$ & $\begin{array}{l}\text { - Envolvimento de alunos de farmácia } \\
\text { de diferentes períodos que tiveram } \\
\text { contato mais próximo com a população, } \\
\text { exercitando sua cidadania e utilizando } \\
\text { conhecimentos adquiridos na academia. }\end{array}$ \\
\hline & \multirow[t]{4}{*}{$\begin{array}{l}\text { Campanha } \\
\text { Dutra na Praça }\end{array}$} & \multirow[t]{4}{*}{$\begin{array}{l}\text { - Em parceria com a Secretaria } \\
\text { Municipal de Saúde de Ribeirão Preto } \\
\text { foram realizados testes, através de } \\
\text { sangue capilar, de glicemia, colesterol, } \\
\text { triglicérides e aferição de pressão arterial } \\
\text { da população adstrita dos bairros } \\
\text { Presidente Dutra e Vila Albertina em } \\
\text { Ribeirão Preto e na Unidade de Saúde } \\
\text { de Família, em datas e locais distintos. } \\
\text { A campanha contou com a participação } \\
\text { de alunos da FCFRP e de funcionários } \\
\text { das UBS Dutra e Vila Albertina. Foram } \\
\text { realizados mais de } 100 \text { testes de } \\
\text { colesterol, cerca de } 150 \text { de glicemia e } \\
\text { aproximadamente } 50 \text { de triglicérides. } \\
\text { Pacientes com resultados muito alterados } \\
\text { foram encaminhados para seguimento na } \\
\text { unidade de saúde de referência. }\end{array}$} & $\begin{array}{l}\text { - Pacientes que tiveram resultados } \\
\text { de glicemia, colesterol e triglicérides } \\
\text { foram acompanhados nas UBS de } \\
\text { referência. Três pacientes com diabetes } \\
\text { descompensado foram referidos ao médico } \\
\text { para insulinização ou otimização da dose } \\
\text { de insulina. Outros três pacientes foram } \\
\text { encaminhados ao médico para otimizar } \\
\text { a dose do antidiabético oral. Pacientes } \\
\text { com hipertrigliceridemia conhecida } \\
\text { encontravam-se com valores elevados, } \\
\text { pois não estavam se tratando devido a } \\
\text { dificuldades em conseguir o medicamento } \\
\text { (genfibrozila). Paciente foi encaminhado } \\
\text { ao médico com orientação para dar } \\
\text { entrada em processo de Alto Custo para } \\
\text { aquisição do medicamento gratuitamente. }\end{array}$ \\
\hline & & & $\begin{array}{l}\text { - Interação entre unidades de saúde, } \\
\text { acadêmicos e docentes das unidades do } \\
\text { campus de Ribeirão Preto - USP }\end{array}$ \\
\hline & & & $\begin{array}{l}\text { - A campanha teve um papel importante } \\
\text { para estreitar ainda mais as ligações da } \\
\text { Universidade com o serviço de saúde. }\end{array}$ \\
\hline & & & $\begin{array}{l}\text { - Acadêmicos de farmácia que participaram } \\
\text { das campanhas candidataram-se a vagas } \\
\text { de estágio junto ao PURAME }\end{array}$ \\
\hline & \multirow[t]{2}{*}{$\begin{array}{l}\text { Campanha } \\
\text { multiprofissional } \\
\text { do NSF-3 }\end{array}$} & \multirow{2}{*}{$\begin{array}{l}\text { - Em parceria com a Secretaria } \\
\text { Municipal de Saúde de Ribeirão Preto } \\
\text { foram realizados testes, através de } \\
\text { sangue capilar, de glicemia, colesterol, } \\
\text { triglicérides e aferição de pressão arterial } \\
\text { da população adstrita. }\end{array}$} & $\begin{array}{l}\text { - Envolvimento de toda a equipe de saúde } \\
\text { das unidades e da comunidade da área do } \\
\text { NSF-3. }\end{array}$ \\
\hline & & & $\begin{array}{l}\text { - Interação entre unidades de saúde, } \\
\text { acadêmicos e docentes das unidades do } \\
\text { campus de Ribeirão Preto - USP }\end{array}$ \\
\hline & $\begin{array}{l}\text { Dia da Saúde: } \\
\text { campanha } \\
\text { uso correto de } \\
\text { medicamentos }\end{array}$ & $\begin{array}{l}\text { - Com o Departamento Municipal de } \\
\text { Saúde de Luiz Antonio foram realizados } \\
\text { testes, através de sangue capilar, de } \\
\text { glicemia, colesterol, triglicérides e } \\
\text { aferição de pressão arterial da população } \\
\text { adstrita, além de orientações sobre } \\
\text { medicamentos. }\end{array}$ & $\begin{array}{l}\text { - Envolvimento dos alunos estagiários do } \\
\text { PURAME com a população do município } \\
\text { que compareceu a esta e outras atividades } \\
\text { realizadas em } 2 \text { locais de saúde do } \\
\text { município. Adultos, jovens e crianças } \\
\text { receberam orientação. }\end{array}$ \\
\hline
\end{tabular}


QUADRO 2

Conjunto de cursos ministrados a alunos de graduação e

profissionais de saúde, inclusive agentes comunitários de saúde

\begin{tabular}{|c|c|c|c|}
\hline TIPO DE AÇÃO & AÇÃO & ATIVIDADES & RESULTADOS \\
\hline \multirow[t]{5}{*}{$\begin{array}{l}\text { Curso sobre } \\
\text { medicamentos }\end{array}$} & $\begin{array}{l}\text { Curso de } \\
\text { prescrição } \\
\text { racional para } \\
\text { acadêmicos } \\
\text { do curso de } \\
\text { medicina da } \\
\text { FMRP-USP }\end{array}$ & $\begin{array}{l}\text { - Curso sobre prescrição racional } \\
\text { de medicamentos foi ministrado, em } \\
\text { conjunto com docentes da FMRP e FCFRP, } \\
\text { aos } 100 \text { alunos do 5o ano de medicina } \\
\text { da FMRP-USP. O curso é baseado em } \\
\text { modelo sugerido pela OMS “Guide for } \\
\text { Good Prescribing” e utiliza o método PBL } \\
\text { (problem based learning) para estruturar } \\
\text { o conceito da prescrição racional. O } \\
\text { curso foi ministrado ao longo do ano } \\
\text { em } 5 \text { vezes, em turmas de } 20 \text { alunos } \\
\text { aproximadamente. }\end{array}$ & $\begin{array}{l}\text { - Cerca de } 100 \text { alunos de medicina } \\
\text { mais conscientes e críticos sobre a } \\
\text { importância da escolha adequada e } \\
\text { racional de medicamentos e tratamentos. } \\
\text { Incorporação de conceitos de assistência } \\
\text { farmacêutica e de uso racional de } \\
\text { medicamentos e do papel da indústria } \\
\text { farmacêutica pelos acadêmicos de } \\
\text { medicina. Avaliação por Objective } \\
\text { Structured Clinical Examination (OSCE). }\end{array}$ \\
\hline & \multirow{2}{*}{$\begin{array}{l}\text { Curso de } \\
\text { prescrição } \\
\text { racional para } \\
\text { profissionais } \\
\text { de saúde do } \\
\text { município de } \\
\text { Luiz Antônio }\end{array}$} & \multirow{2}{*}{$\begin{array}{l}\text { - Utilizando o modelo do curso } \\
\text { ministrado aos alunos de medicina, } \\
\text { com algumas alterações, ministrou- } \\
\text { se o curso "Prescrição Racional de } \\
\text { Medicamentos" para os profissionais de } \\
\text { saúde do município de Luiz Antônio, como } \\
\text { estratégia para melhorar a utilização de } \\
\text { medicamentos nessa localidade. }\end{array}$} & $\begin{array}{l}\text { - Compreensão pelos profissionais dos } \\
\text { processos que devem nortear uma boa } \\
\text { prescrição médica ou odontológica. }\end{array}$ \\
\hline & & & $\begin{array}{l}\text { - Interação da equipe de saúde de Luiz } \\
\text { Antônio. }\end{array}$ \\
\hline & \multirow{2}{*}{$\begin{array}{l}\text { Curso sobre } \\
\text { "uso racional de } \\
\text { medicamentos" } \\
\text { para agentes } \\
\text { comunitários } \\
\text { de saúde }\end{array}$} & \multirow{2}{*}{$\begin{array}{l}\text { - Curso sobre uso racional de } \\
\text { medicamentos foi ministrado a } 17 \\
\text { agentes comunitários de saúde (ACS) de } \\
3 \text { equipes de saúde da família do bairro } \\
\text { Presidente Dutra e a } 4 \text { ACS mais cerca de } \\
10 \text { profissionais de saúde. Para o curso, } \\
\text { utilizou-se o material disponibilizado } \\
\text { pelo Ministério da Saúde, intitulado } \\
\text { "Uso Racional de Medicamentos”. }\end{array}$} & $\begin{array}{l}\text { - Estreitamento das relações dos agentes } \\
\text { comunitários da saúde e de outros } \\
\text { profissionais com a equipe de farmácia. }\end{array}$ \\
\hline & & & $\begin{array}{l}\text { - Agentes comunitários de saúde e } \\
\text { técnicos treinados para auxiliarem na } \\
\text { educação da comunidade sobre uso } \\
\text { adequado de medicamentos, contribuindo } \\
\text { para a redução de erros e problemas com } \\
\text { medicamentos. }\end{array}$ \\
\hline
\end{tabular}

\section{QUADRO 3}

Gerenciamento de medicamentos obtidos e distribuídos pela rede municipal para aprimoramento do acesso da população aos medicamentos nos municípios de Luiz Antonio e Ribeirão Preto - SP

\begin{tabular}{|c|c|c|c|}
\hline TIPO DE AÇÃO & AÇÃO & ATIVIDADES & RESULTADOS \\
\hline \multirow[t]{3}{*}{$\begin{array}{l}\text { Gerenciamento } \\
\text { de } \\
\text { medicamentos }\end{array}$} & \multirow[t]{3}{*}{$\begin{array}{l}\text { Aquisição, } \\
\text { estoque e } \\
\text { dispensação de } \\
\text { medicamentos }\end{array}$} & $\begin{array}{l}\text { - Coleta de medicamentos provenientes } \\
\text { de doações da comunidade, de médicos } \\
\text { das unidades e de laboratórios } \\
\text { farmacêuticos. Os medicamentos } \\
\text { vencidos também eram recolhidos para } \\
\text { destino devido. }\end{array}$ & $\begin{array}{l}\text { - Acesso de pacientes das unidades } \\
\text { assistidas aos medicamentos } \\
\text { padronizados e não padronizados na } \\
\text { Rede Pública Municipal de Saúde. }\end{array}$ \\
\hline & & $\begin{array}{l}\text { - Organização de medicamentos } \\
\text { classificados e cadastrados no } \\
\text { computador. }\end{array}$ & \multirow{2}{*}{$\begin{array}{l}\text { - Destinação adequada de } \\
\text { medicamentos vencidos que seriam } \\
\text { jogados no lixo comum nos domicílios } \\
\text { dos pacientes. }\end{array}$} \\
\hline & & $\begin{array}{l}\text { - Organização de planilha de estoque } \\
\text { para controle de entrada/saída de } \\
\text { medicamentos. }\end{array}$ & \\
\hline
\end{tabular}


QUADRO 4

Descrição das Práticas de Farmácia Clínica realizadas junto a unidades de saúde nos municípios de Luiz Antonio e Ribeirão Preto, atividades e resultados obtidos

\begin{tabular}{|c|c|c|c|}
\hline TIPO DE AÇÃO & AÇÃo & ATIVIDADES & RESULTADOS \\
\hline \multirow{7}{*}{$\begin{array}{l}\text { Práticas de } \\
\text { farmácia } \\
\text { clínica }\end{array}$} & \multirow{2}{*}{$\begin{array}{l}\text { Orientação e } \\
\text { acompanhamento } \\
\text { de pacientes }\end{array}$} & $\begin{array}{l}\text { - Avaliação de prescrição de pacientes e } \\
\text { intervenção nas prescrições inadequadas. }\end{array}$ & \multirow{2}{*}{$\begin{array}{l}\text { - Muitos pacientes estão polimedicados, } \\
\text { com excesso de prescrição ou até dupla } \\
\text { prescrição. Alguns dos problemas } \\
\text { podem ser detectados no momento da } \\
\text { orientação sobre o uso de medicamentos. } \\
\text { A confecção de cartões que contém todos } \\
\text { os medicamentos que o paciente usa de } \\
\text { forma conciliada, instrumentalizando } \\
\text { o prescritor e facilitando o trabalho } \\
\text { do farmacêutico pode contribuir para } \\
\text { identificar problemas e erros. }\end{array}$} \\
\hline & & $\begin{array}{l}\text { - Monitoramento de glicemia de } \\
\text { pacientes diabéticos e de pressão arterial } \\
\text { de pacientes hipertensos. }\end{array}$ & \\
\hline & $\begin{array}{l}\text { Prescrição } \\
\text { informatizada }\end{array}$ & $\begin{array}{l}\text { - Desenvolvimento de software para } \\
\text { prescrição informatizada com recursos } \\
\text { do CNPq. }\end{array}$ & $\begin{array}{l}\text { - Criação do SIPPFT - sistema } \\
\text { informatizado de prescrição e } \\
\text { seguimento farmacoterapêutico. }\end{array}$ \\
\hline & \multirow{4}{*}{$\begin{array}{l}\text { Comissão de } \\
\text { medicamentos e } \\
\text { terapêutica do } \\
\text { município de } \\
\text { Luiz Antônio }\end{array}$} & $\begin{array}{l}\text { - Análise quanti e qualitativa dos } \\
\text { medicamentos distribuídos pela rede } \\
\text { pública do município de Luiz Antônio. }\end{array}$ & $\begin{array}{l}\text { - Lista padronizada atualizada } \\
\text { periodicamente. }\end{array}$ \\
\hline & & $\begin{array}{l}\text { - Revisão dos processos de compra de } \\
\text { medicamentos. }\end{array}$ & $\begin{array}{l}\text { - Maior conhecimento sobre a } \\
\text { assistência farmacêutica do município. }\end{array}$ \\
\hline & & $\begin{array}{l}\text { - Reuniões sistemáticas para discutir } \\
\text { a utilização de medicamentos, a } \\
\text { padronização ou a exclusão de produtos } \\
\text { farmacêuticos da lista padronizada. }\end{array}$ & \multirow{2}{*}{$\begin{array}{l}\text { - Resultados de melhoria de } \\
\text { gerenciamento da assistência } \\
\text { farmacêutica não foram alcançados } \\
\text { pela deficiência técnica do farmacêutico, } \\
\text { não resultando em redução significativa } \\
\text { de gastos e melhor suprimento de } \\
\text { medicamentos para a população. } \\
\text { Esforços do gestor e apoio técnico } \\
\text { são constantes para tentar reverter } \\
\text { este quadro. }\end{array}$} \\
\hline & & $\begin{array}{l}\text { - Estudos para avaliação técnica da } \\
\text { utilização de medicamentos. }\end{array}$ & \\
\hline
\end{tabular}

